\title{
Análisis bibliométrico de la producción científica de las universidades estatales de Costa Rica indexadas en Scopus, 2011-2019: una aplicación con el paquete de lenguaje $\mathbf{R}$ "Bibliometrix" *
}

Bibliometric Analysis of the Scientific Production of Costa Rican State Universities Indexed in Scopus, 2011-2019: an Application with the R Language Package "Bibliometrix"

Silvia Sáenz León

Consejo Nacional de Rectores, Costa Rica ORCID: https://orcid.org/0000-0003-0801-8224

Nanci Rodríguez Ramos

Consejo Nacional de Rectores, Costa Rica ORCID: https://orcid.org/0000-0002-7848-4434

Recibido: 05 de abril de 2021

Aceptado: 13 de agosto de 2021

Publicado: 24 de enero de 2022

\section{Resumen:}

El presente artículo está orientado al análisis de algunos indicadores bibliométricos, aplicados a la producción científica realizada por las universidades estatales de Costa Rica para el período 2011-2019. Mediante el lenguaje en R y el uso de la aplicación Biblioshiny de la librería/paquete Bibliometrix, se generó una serie de indicadores relacionados con la productividad por autor, afiliación, nivel de citación, entre otras características documentales, consumo científico e impacto.

Entre los principales hallazgos se evidencia que la mayor parte de la producción científica de Costa Rica, indexada en Scopus, proviene de las universidades estatales; hay presencia de la investigación costarricense en todos los continentes y no únicamente como participantes, sino como líderes o investigadores principales; existe gran diversificación de publicación en revistas de múltiples disciplinas; y hay diversidad de investigación en múltiples áreas de la ciencia, entre otros.

Palabras claves: Bibliometría, Indicadores, Actividad científica.

\section{Abstract:}

This article is oriented to the analysis of some bibliometric indicators, applied to the scientific production by the state universities of Costa Rica for the period 2011-2019. Through the $\mathrm{R}$ language and the use of the Biblioshiny application from the Bibliometrix 
library / package, a series of indicators related to productivity by author, affiliation, level of citation, documentary characteristics, scientific consumption, and impact.

Among the main findings, it is evident that most of the scientific production of Costa Rica indexed in Scopus comes from state universities, there is a presence of Costa Rican research in all continents not only as participants as leaders or main researchers, but there is also great diversification of publication in journals of multiple disciplines, diversity of research in multiple areas of science and others.

Keywords: Bibliometric, Indicators, Scientific activity.

\section{Introducción}

El presente documento aborda el análisis de algunos indicadores bibliométricos, obtenidos con base en la información referencial de las publicaciones que cuentan con afiliación de las universidades estatales de Costa Rica, indizada en la base de datos internacional Scopus para los años 2011-2019. La obtención de estos indicadores se logró mediante el paquete bibliométrico "Bibliometrix" con su aplicación "Biblioshiny", los cuales se ejecutan mediante el lenguaje de programación $\mathrm{R}$ de código abierto.

El objetivo principal de este estudio es ofrecer un análisis basado en la producción científica de las universidades estatales de Costa Rica, indexada en Scopus, para conocer a profundidad sus características tomando en cuenta la tipología documental, productividad científica, revistas indexadas y algunos otros indicadores que tienen que ver con el consumo científico e impacto de la investigación a nivel de citación y visualizaciones. Para finalmente, a raíz este análisis generalizado, brindar algunos hallazgos y recomendaciones relevantes que busquen fortalecer la actividad investigativa.

\section{Aproximación conceptual}

El origen histórico de los análisis bibliométricos es difícil de situar en el tiempo, no obstante, de acuerdo con Sanz y Martín (1997) existe literatura sobre el tema que deriva incluso desde los años treinta; a raíz de la necesidad de contar con información sobre el rumbo de la ciencia en esos años. El objetivo principal de los estudios bibliométricos es, en términos generales, estudiar la producción científica divulgada en medios formales. Estos análisis hacen uso de 
distintos indicadores obtenidos a partir de información o metadatos referenciales. Muchas veces se le denomina a la bibliometría "la ciencia de la ciencia" según indica Uceda (1997).

A lo largo de la historia se han realizado varios estudios de carácter bibliométrico como el de Lomonte y Ainsworth (2002); Nielsen et al. (2012); Alarcon et al. (2019); Duran et al. (2019); Yılmaz et al. (2019), donde se generan y analizan principalmente estadísticos descriptivos para la toma de decisiones basados en la variabilidad de documentos, autores más productivos, medición de los cambios en la producción científica, obsolescencia y actualidad de diversos campos de la ciencia; por ejemplo, el campo de la nanotecnología o de la literatura presente sobre el accidente cerebrovascular. También se analizó el comportamiento de los cuartiles de impacto y de las búsquedas o citaciones de buscadores como Google Scholar.

La bibliometría está vinculada con la evaluación de las actividades generadoras de I+D. En este proceso, por lo general, se consideran cuatro fases importantes: la entrada de recursos (inputs) destinados a este tipo de actividades; la transformación de estos recursos en investigación (proceso); los productos que se generan (outputs) y, por último, años después de la publicación, el impacto (consumo científico). La bibliometría se ubica dentro de la medición de productos y puede dar cuenta del impacto en términos de citación, además, está directamente relacionada con la productividad de los investigadores señala Cortés (2007).

En algunas ocasiones, suele hablarse de cienciometría o bibliometría indistintamente; sin embargo, la cienciometría es la disciplina que aborda tanto la bibliometría como la informetría ${ }^{1}$. Según se plantea en Lascurain (2006), este tipo de estudios puede tener un alcance micro, meso y macro. El primer nivel (micro) toma en cuenta los datos referenciales sobre publicaciones de un artículo en particular, el alcance meso considera un conjunto de publicaciones atribuidas a una subdisciplina.

Finalmente, el nivel macro considera las publicaciones que forman parte de una disciplina en su conjunto, o un bien, toma en cuenta un grupo de publicaciones a nivel regional e 
internacional. Los niveles de desagregación y lo que plantea Urbizagástegui (2014), sobre el uso de la cienciometría y bibliometría, han facilitado el desarrollo de diversas métricas para estudiar la productividad de los investigadores, la obsolescencia de la literatura científica, el auge de algunas disciplinas y subdisciplinas, entre otros temas de gran interés que forman parte de las características de la ciencia y sus constantes cambios.

La bibliometría tiene entonces, como núcleo de estudio, la información derivada de las publicaciones, consideradas como todos aquellos documentos difundidos mediante canales formales, por ejemplo: artículos de revistas, informes técnicos, ponencias, patentes, libros, entre otros. Las revistas en sí mismas constituyen un sujeto de estudio importante para la bibliometría, ya que son el medio usual para divulgar el conocimiento científico y, a su vez, es validado por la comunidad científica internacional, expone Sancho (2003).

Un insumo valioso de la bibliometría son los indicadores y métricas que permite obtener, de acuerdo con Spinak (1998), este tipo de indicadores a partir de los cuales se hacen varios tipos de comparación con el fin de respaldar una evaluación y monitoreo de la investigación. Como indicadores clave se destacan el número total de publicaciones, el total de afiliaciones (colaboración internacional/nacional), disciplinas o áreas de la ciencia, idiomas de publicación y otras clasificaciones de tipo documental, relacionados muchas veces con el crecimiento y, que generalmente, se expresan en términos longitudinales o de series de tiempo.

Otro tipo de indicadores destacados en la bibliometría son los relacionados con el consumo científico, los cuales abordan la citación y el uso del conocimiento. Algunos de estos indicadores tienen que ver con el recuento de citas, de acuerdo con Gómez y Bordons (2009, p.795) se entiende como "el análisis de citas conlleva el recuento del número de citas que reciben los documentos durante un determinado período de tiempo después de su publicación". Ahora bien, se debe tener algunas consideraciones con este tipo de indicadores a la hora de realizar comparaciones, por ejemplo, el hecho de comparar el nivel de citación de un investigador novel ante el nivel de citación de un investigador muy experimentado, ya que puede ser muy distinto y en algunos casos poco objetivo. 
En cuanto al impacto, Hirsch $(2005 ; 2010)$ crea del índice $\mathrm{H}$, el cual consiste en el número $\mathrm{n}$ de publicaciones citadas al menos $\mathrm{n}$ veces en un período de referencia. Para su cálculo se ordenan y enumeran las publicaciones por su número de citas recibidas en forma descendente, el punto en el que el número de orden coincide con el de citación constituye el índice. Este indicador se utiliza para calificar el impacto de un científico mediante una sola cifra, sin embargo, nunca debe confundirse con un indicador de calidad según plantea Montazerian et al. (2020).

Actualmente, el índice $\mathrm{H}$ es ampliamente reconocido como un indicador que complementa la evaluación de la investigación y como un indicador del consumo científico, aunque también se le ha atribuido algunas consideraciones como, por ejemplo, cuando se utiliza para realizar comparaciones; no es lo mismo un índice $\mathrm{h}$ de un investigador experimentado, a uno que recién comienza su carrera de publicación. Por su parte, el número de citas es otro indicador bastante utilizado y puede aproximar la visibilidad y el prestigio de un investigador en la comunidad científica.

Sea cual sea el enfoque de indicadores bibliométricos a utilizar (de tipología documental o consumo e impacto), existen diversos softwares y aplicaciones en especial de código abierto que facilitan la obtención y análisis de este tipo de métricas. Se destaca para esta investigación el uso del lenguaje informático estadístico R mediante la biblioteca "Bibliometrix". Esta biblioteca de acceso abierto permite importar datos de diversos índices como Scopus, Web of Science, PudMed, entre otros (Aria y Curccurullo, 2017).

A partir de su versión 2.0 se introduce a "Bibliometrix" la aplicación "Biblioshiny", la cual facilita el uso, procesamiento y análisis de los metadatos ya que se realiza a través de una interfaz en el navegador. El análisis se divide en tres grandes procesos, descritos a continuación (ver Figura 1):

1. Recolección de datos: se extraen los distintos metadatos. 
2. Análisis de datos, articulado en tres subetapas:

2.1. Análisis descriptivo de los datos bibliográficos: devuelve las principales características como idioma, tipo de documento, conteos generales, entre otros.

2.2. Generación de redes de análisis de acoplamiento bibliográfico: en esta fase básicamente se generan indicadores compuestos de atributos conectados, por ejemplo, hay autores que se relacionan con otros, países en colaboración, temáticas relacionadas, entre otros.

2.3. Normalización: se normalizan los datos de acoplamiento bibliográfico, es decir, se calcula una medida de similitud.

3. Visualización de datos: se puede visualizar los datos o redes obtenidas con representaciones gráficas.

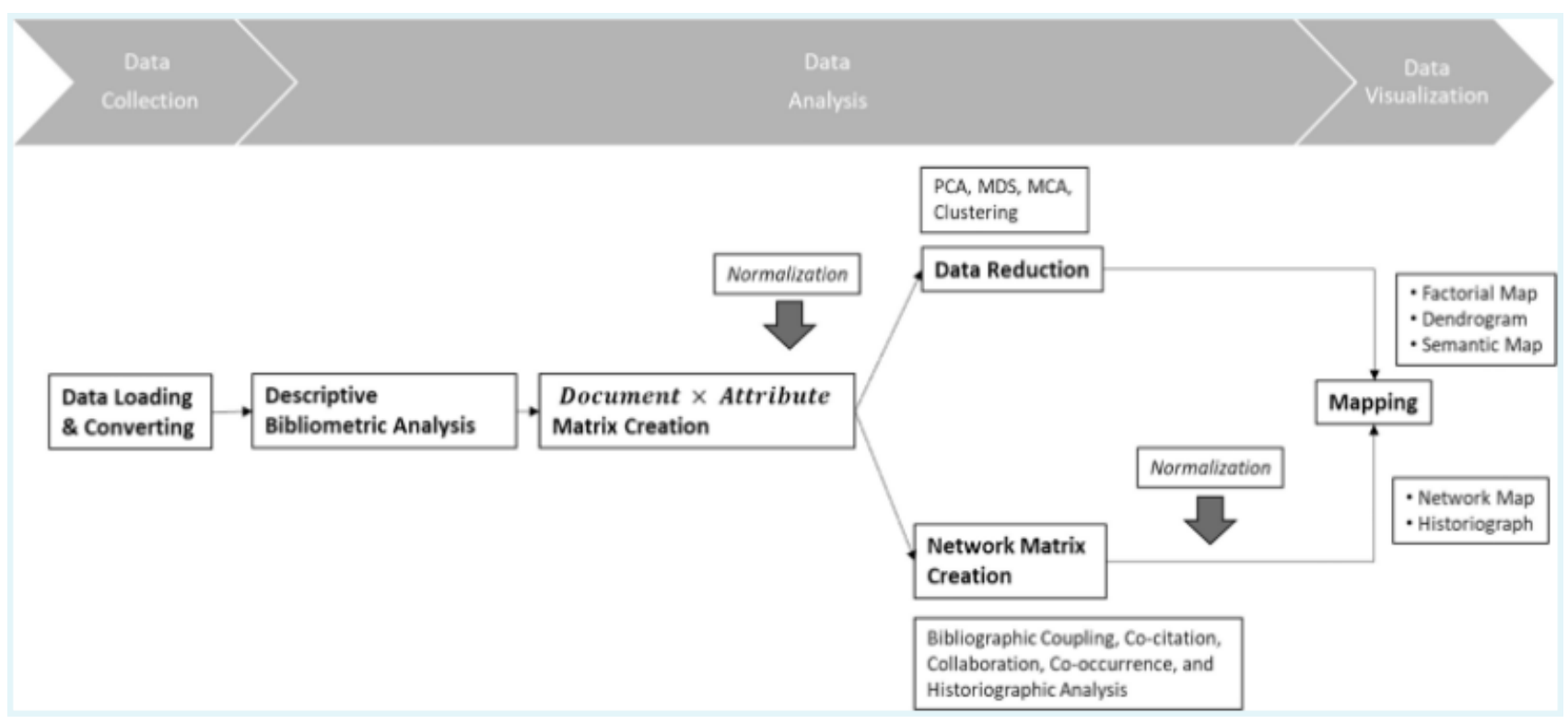

Figura 1. Mapa de procesos de Biblioshiny

Fuente: Tomado de (Aria y Curccurullo, 2017, p. 936).

\section{Materiales y método}

Para este trabajo se utilizó la información referencial de todas aquellas publicaciones indexadas en la base de datos Scopus, realizadas por las universidades estatales de Costa Rica durante los años 2011-2019. 
Para lo cual se definió la siguiente sintaxis de búsqueda:

"AFFILCOUNTRY ( costa AND rica) AND ( LIMIT-TO ( PUBYEAR, 2019 ) OR LIMIT-

TO ( PUBYEAR, 2018 ) OR LIMIT-TO (PUBYEAR, 2017 ) OR LIMIT-

TO ( PUBYEAR, 2016 ) OR LIMIT-TO ( PUBYEAR, 2015 ) OR LIMIT-

TO ( PUBYEAR, 2014 ) OR LIMIT-TO ( PUBYEAR, 2013 ) OR LIMIT-

TO ( PUBYEAR, 2012 ) OR LIMIT-TO ( PUBYEAR, 2011 )) AND ( LIMIT-TO ( AF-

ID , "Universidad de Costa Rica" 60071929 ) OR LIMIT-TO ( AF-ID , "Universidad Nacional de Costa Rica" 60071924 ) OR LIMIT-TO ( AF-ID , "Tecnológico de Costa Rica,

Cartago" 60099123) OR LIMIT-TO ( AF-ID , "Universidad Estatal a Distancia" 60113197$)$ )"

Con un registro de 5.741 documentos se realizó la exportación de los datos en formato csv ${ }^{2}$ durante el mes de setiembre del año 2020. Cabe destacar que en el caso de la Universidad Técnica Nacional (UTN), que recién está desarrollando sus campos de investigación, no se contabiliza aún como institución en los filtros de búsqueda de la base de datos. Por lo anterior, se solicitó información de sus publicaciones de manera directa a la Vicerrectoría de Investigación y, según este reporte, la producción de esta universidad fue de dos documentos: uno publicado en el año 2016 y otro en el año 2018 (G. Ramírez, comunicación personal, 2020); no obstante, estas publicaciones no se incluyeron en la generación de indicadores por la poca variabilidad que presenta. A partir de las descargas de los datos se obtuvieron indicadores como la productividad por autor, publicaciones por año, medios en los que se publica, idioma y tipo documental, colaboración internacional, citación e impacto. A continuación, se detalla el proceso a seguir:

El primer paso es descargar la librería "Bibliometrix" en la consola de comandos de "RStudio", con la siguiente función:

$$
\text { > install.packages("bibliometrix") }
$$

De forma seguida se abre la aplicación "Biblioshiny" con la función:

$$
\text { > biblioshiny() }
$$


Con este procedimiento se abre la interfaz en el navegador que permite importar los datos de la base de datos referencial de interés. Posterior a la importación de los metadatos, se procedió con el análisis descriptivo, mediante la función "biblioAnalysis", la cual calcula las principales medidas bibliométricas utilizando la sintaxis [results <- biblioAnalysis(M, sep = ";")], ello devuelve datos relacionados con características documentales básicas, como tipo de documento, citación total, documentos por año, entre otros (Aria y Curccurullo, 2017).

Tal y como se detalló en el apartado conceptual, la aplicación entre sus procesos también genera información relacionada con el impacto al considerar el índice $\mathrm{H}$ por autor, por revista y el consumo científico mediante el conteo de citaciones. Desde la aplicación se puede obtener diversos gráficos, no obstante, algunos son de baja calidad visual, no editables y no reconocen las tildes o caracteres especiales incluidos en los nombres propios de las personas investigadoras o de las universidades o centros firmantes en la afiliación. Por este motivo los análisis resultantes se exportaron y se graficaron con el programa Excel para su correcta visualización.

Para el análisis de las revistas costarricenses indexadas en Scopus, se exportó información sobre su factor de impacto SJR en el portal SClmago Journal and Country Rank, en las distintas disciplinas en las cuales se desempeña cada revista, a partir de esta información, se procedió a obtener su posición en cuartiles, año de indexación, tipo de acceso, entre otros.

\section{Principales resultados}

Es este apartado se expondrán los principales resultados del cálculo de indicadores relevantes que consideran la producción científica costarricense realizada en las universidades estatales, indexada en el índice internacional Scopus.

\section{a. Características relevantes de las revistas costarricenses indexadas en Scopus}

A la fecha, existen seis revistas indexadas en la base de datos Scopus, pertenecientes a dos universidades estatales de Costa Rica (Universidad de Costa Rica (UCR) y Universidad Nacional (UNA)). A modo de síntesis se presenta a continuación (ver Cuadro 1) algunas 
características de las revistas mencionadas, considerando su afiliación, fecha de indexación, tipo de acceso y cuartil de impacto (según el SClmago Journal and Country Rank). Es importante mencionar que las revistas que cuentan con una indexación muy reciente aún no poseen, de forma exacta, su posición en los cuartiles de impacto del Ranking.

\section{Cuadro 1}

Características de las revistas costarricenses indexadas en la base de datos Scopus

\begin{tabular}{|c|c|c|c|c|c|}
\hline Revista & Universidad & $\begin{array}{c}\text { Fecha de } \\
\text { indexación }\end{array}$ & $\begin{array}{c}\text { Tipo de } \\
\text { acceso }\end{array}$ & Disciplina científica & $\begin{array}{c}\text { Cuartil de } \\
\text { impacto }\end{array}$ \\
\hline $\begin{array}{c}\text { Revista Biología } \\
\text { Tropical }\end{array}$ & UCR & 1969 & Abierto & $\begin{array}{c}\text { Ciencias Biológicas y } \\
\text { Agrícolas }\end{array}$ & Q2 \\
$\begin{array}{c}\text { Revista } \\
\text { Rnkesteriana }\end{array}$ & UCR & $\begin{array}{c}2004-2007, \\
2013-2019\end{array}$ & Abierto & $\begin{array}{c}\text { Ciencias Biológicas y } \\
\text { Agrícolas (revista } \\
\text { especializada en } \\
\text { orquídeas) }\end{array}$ & Q2 \\
\hline $\begin{array}{c}\text { Electrónica } \\
\text { Educare }\end{array}$ & UNA & 2017 & Abierto & Educación & Q3 \\
\hline $\begin{array}{c}\text { Revista Agronomía } \\
\text { Mesoamericana }\end{array}$ & UCR & $2019-2020$ & Abierto & $\begin{array}{c}\text { Ciencias Biológicas y } \\
\text { Agrícolas }\end{array}$ & NA \\
\hline Revista Uniciencia & UNA & $2019-2020$ & Abierto & $\begin{array}{c}\text { Multidisciplinaria: } \\
\text { biología, física, } \\
\text { informática, } \\
\text { matemáticas, química, } \\
\text { topografía y geodesia }\end{array}$ & NA \\
\hline $\begin{array}{c}\text { MHSalud } \\
\text { UnA }\end{array}$ & $2019-2020$ & Abierto & $\begin{array}{c}\text { Bultidisciplinaria: } \\
\text { biología molecular, } \\
\text { ortopedia y medicina } \\
\text { del deporte }\end{array}$ & NA \\
\hline
\end{tabular}

Fuente: Elaboración propia con datos de Scopus y del SCImago Journal and Country Rank (2020). 


\section{b. Características documentales de la producción científica costarricense indexada en Scopus 2011-2019}

Las publicaciones científicas de las universidades estatales de Costa Rica indexadas en la base internacional Scopus han experimentado un índice de crecimiento anual del $13 \%$, en términos generales, presentan una tendencia creciente con una leve disminución en el año 2013 de un $-1 \%$ y en el año 2015 de un -4\%. A continuación, en la Figura 2 se presenta la trayectoria del total de las publicaciones actualizadas al año 2020 realizadas por las universidades estatales de Costa Rica en el período 2011-2019.

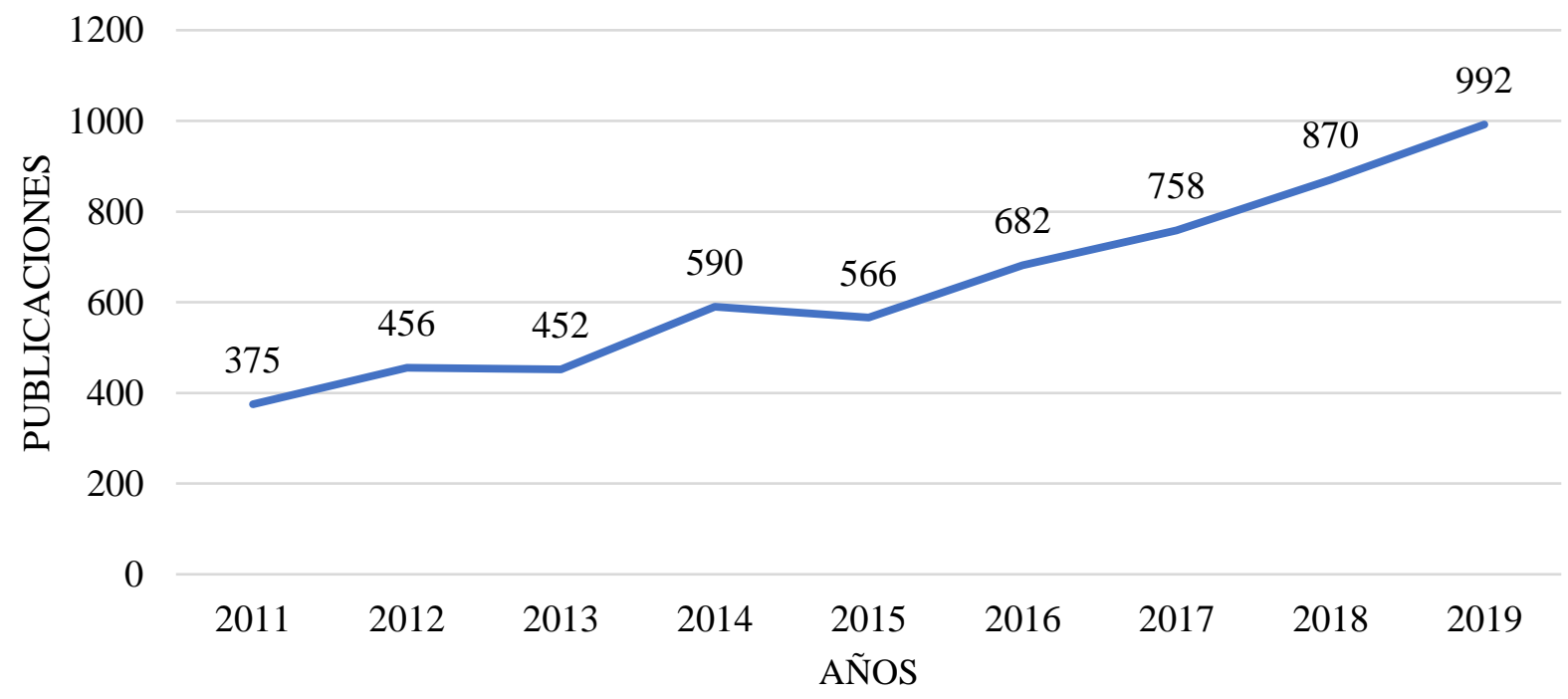

Figura 2. Total de las publicaciones de las universidades públicas de Costa Rica indexadas en la base de datos de Scopus, 2011-2019

Fuente: Elaboración propia con datos descargados de Scopus 2011-2019.

Sobre los medios en los cuales se publica, se muestran cierta diversificación y aumento al igual que el comportamiento mostrado en las publicaciones. Estos medios van desde revistas, libros, conferencias, entre otros. En la Figura 3, se observa la tendencia que han seguido los distintos medios de publicación mencionados, únicamente se reporta una caída de -3\% en el año 2015. 


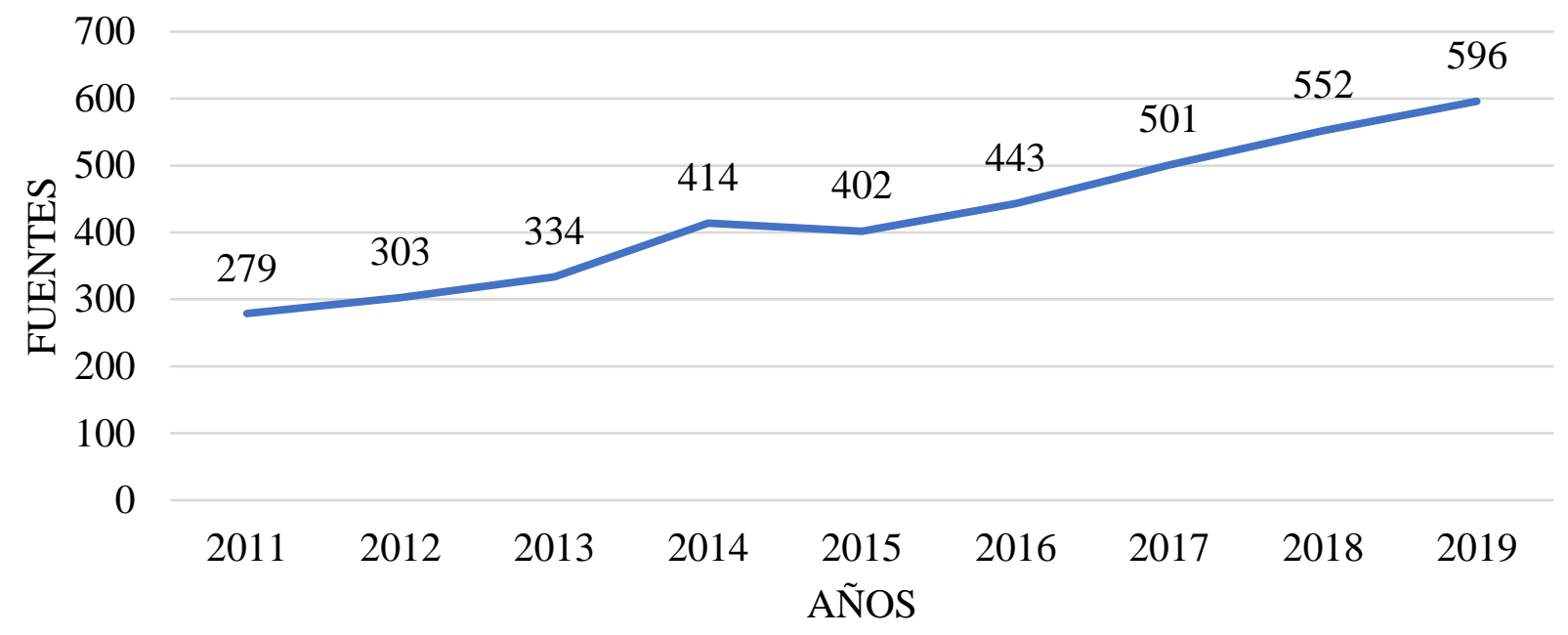

Figura 3. Total de medios en los que publican las universidades estatales de Costa Rica, 2011-2019

Fuente: Elaboración propia con datos tomados de Scopus 2011-2019.

En cuanto al aporte porcentual de las universidades estatales con respecto al total de publicaciones científicas costarricenses indexadas en Scopus, las universidades concentran la mayor proporción. En promedio para el período en estudio las publicaciones de las universidades estatales han contribuido con el $66 \%$ del total de publicaciones en este índice.

En la Figura 4 se presenta la distribución de las publicaciones científicas indexadas en Scopus con afiliación de las universidades estatales y con afiliación de otras organizaciones de Costa Rica. De igual forma que el total de publicaciones, la proporción ha crecido al 2019, la contribución de las universidades se ha situado en el $73 \%$ con respecto al resto de producción.

Con respecto a la productividad por autor, de acuerdo con un top 20 de autores analizados, se destaca la contribución de José María Gutiérrez investigador de la Universidad de Costa Rica (UCR), quien figura en la primera posición y cuya producción de artículos se ubica en 22 documentos científicos en promedio por año. En el segundo lugar se ubica el investigador Bruno Lomonte de la UCR con una producción promedio por año de 13 artículos y en el tercer 
puesto, se encuentra el investigador Roger Moya del Instituto Tecnológico (TEC) con un promedio de 10 artículos por año.

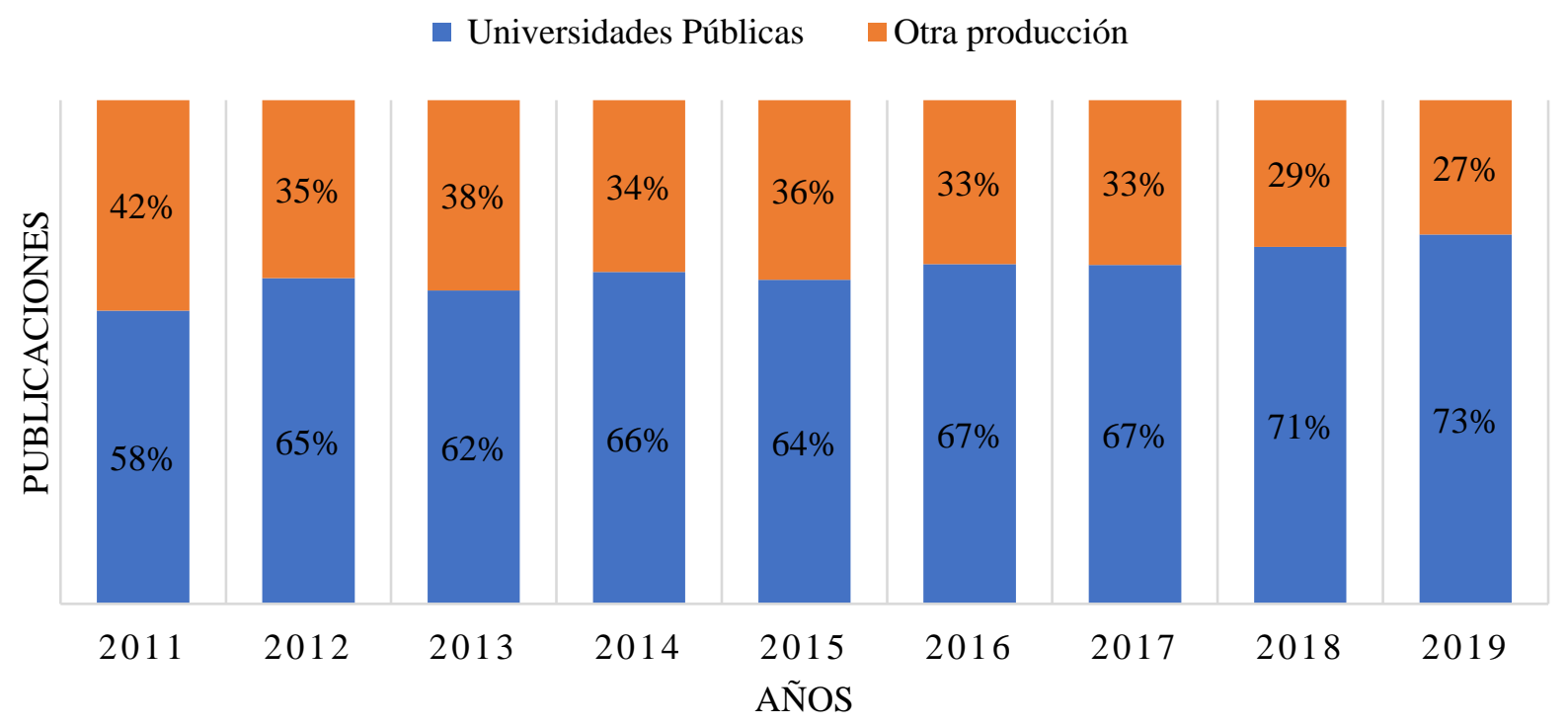

Figura 4. Distribución de las publicaciones indexadas en Scopus realizadas por las universidades estatales y otra producción nacional, 2011-2019

Fuente: Elaboración propia con datos de Scopus (2011-2019).

Dentro de este top y específicamente en los primeros ocho lugares se destaca el aporte de dos autores extranjeros Juan Calvete y Ramón Vilanova, ambos investigadores procedentes de España con 7 y 8 documentos en promedio respectivamente, estos autores demuestran gran colaboración y vinculación con Costa Rica. En el séptimo lugar destaca el investigador Luis Fernando Chaves de la UNA con un promedio de 6 artículos anuales y finalmente, el investigador Guillermo León de la UCR con 6 documentos en promedio.

A continuación, se presenta en la Figura 5 un top 20 de los autores más productivos para el período de referencia, cuya publicación está vinculada a las universidades estatales para el período analizado. 


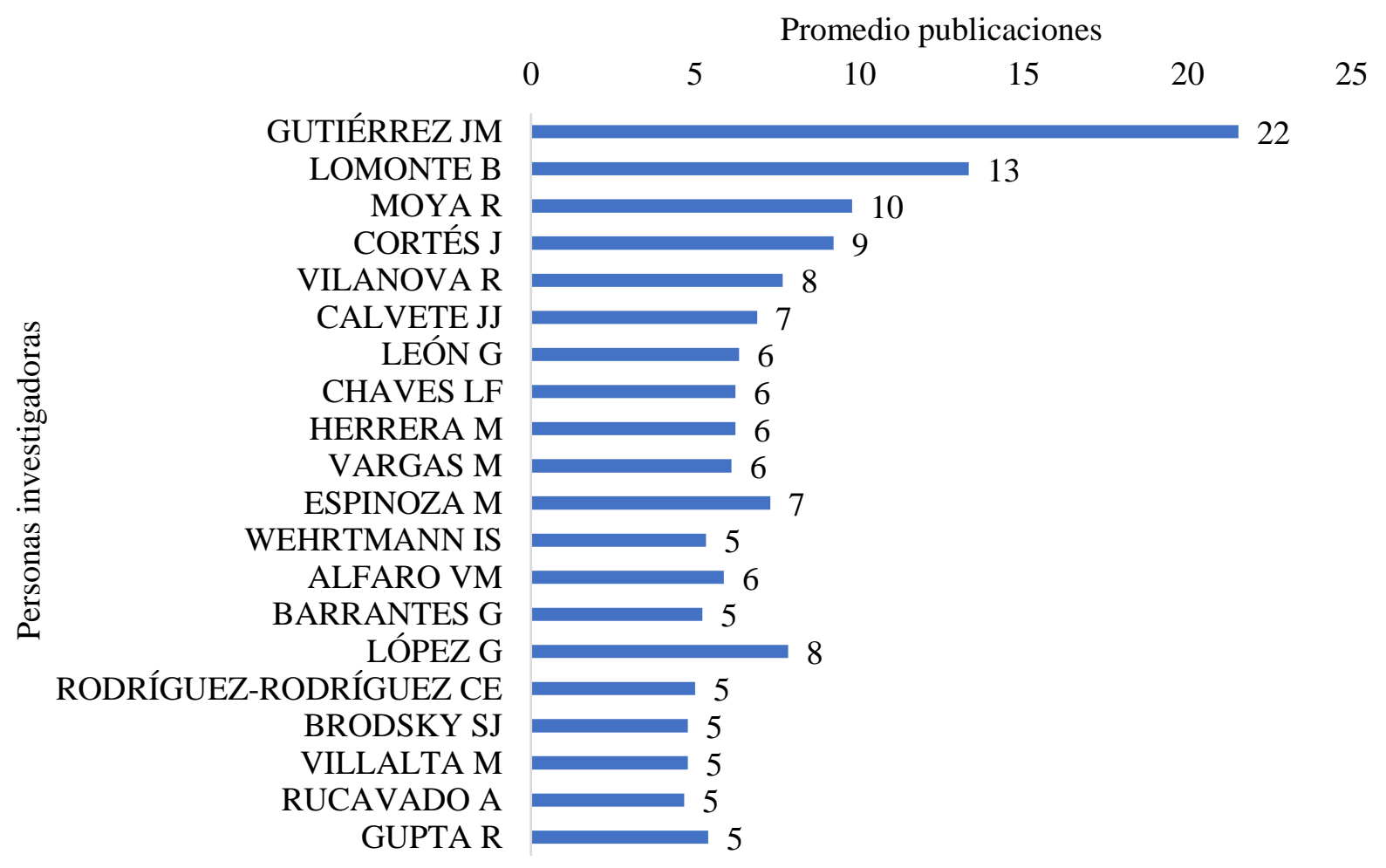

Figura 5. Promedio de publicaciones anuales de los Investigadores (top 20) con mayor número de publicaciones, 2011-2019

Fuente: Elaboración propia con datos de Scopus.

En términos generales el producto científico que más se publica por parte de las universidades estatales costarricenses, son artículos originales que corresponden a un $74,8 \%$ del total de la producción científica para el período de referencia, las ponencias concentran un 14,3\% de las publicaciones, y, en tercer lugar, los review o artículos de revisión teórica los cuales concentran un $4,6 \%$. También se destacan los capítulos de libro con un $3,7 \%$ de las publicaciones realizadas, en menor medida se registran otro tipo de documento como cartas, erratas, notas, entre otros (ver Figura 6). 


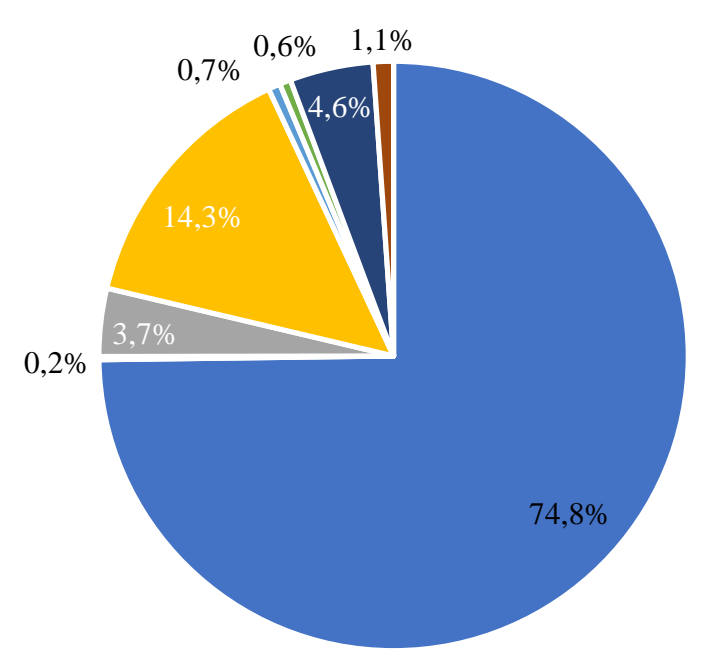

- Artículo

- Libro

- Capítulo de libro

- Ponencia

$\square$ Editorial

- Cartas

- Artículo de revisión

- Otros

Figura 6. Distribución de las publicaciones por tipo de documento, (total 2011-2019) Fuente: Elaboración propia con datos descargados de Scopus, 2011-2019.

Referente a los idiomas de publicación, de acuerdo con los datos analizados, se publicaron en total 4.968 documentos en inglés y 617 documentos en idioma español, estos dos idiomas concentran la mayoría de las publicaciones realizadas. Tal como se puede observar en la figura 7 , tanto las publicaciones en inglés y en español han aumentado. No obstante, la brecha aún sigue siendo muy marcada con preponderancia del idioma inglés.

Además, se publicó un documento en idioma alemán, ruso y japonés, 14 publicaciones en portugués y 4 en francés. También hay 134 publicaciones en dos o más idiomas simultáneamente, de estas 134 publicaciones, 113 son en inglés y español. 


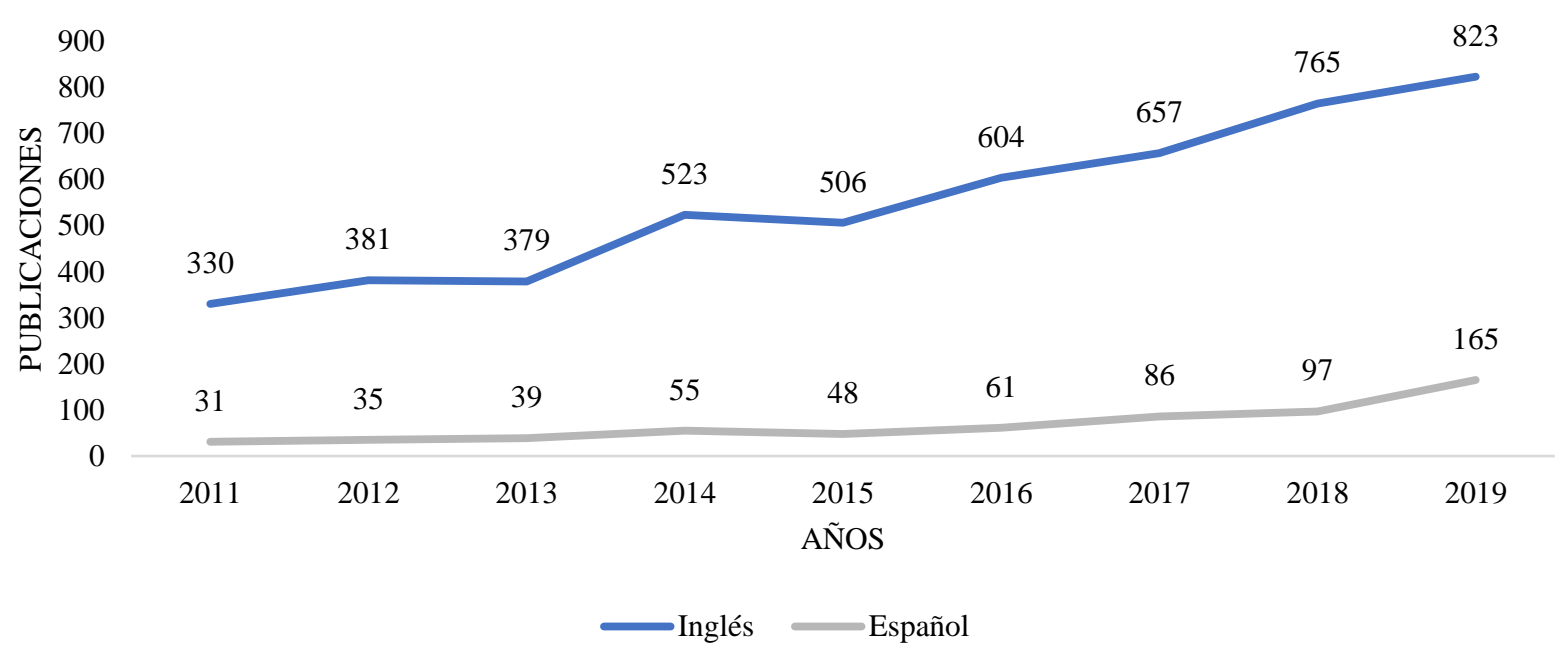

Figura 7. Total de publicaciones indexadas por tipo de idioma (top 2), 2011-2019 Fuente: Elaboración propia con datos de SCOPUS (2011-2019).

Referente a la colaboración internacional, de acuerdo con la publicación en términos de autoría principal, Costa Rica encabeza el listado con 1.224 documentos en los cuales un investigador de las universidades estatales costarricenses figura como investigador líder 0 coordinador, esto se traduce en un $46,9 \%$ del total de las publicaciones analizadas. En segundo lugar, se ubica Estados Unidos con un 13,6\% de colaboración equivalente a 355 publicaciones, seguido de España que cuenta con autoría principal en 221 publicaciones lo que representa un 8,5\%. Entre los países con menor autoría de este top se ubican Dinamarca e Israel con 16 documentos que representan un $0,6 \%$ y Suecia con 14 documentos que concentran el $0,5 \%$ de la producción científica total analizada.

A continuación, se presenta en la figura 8 la distribución de los países que han colaborado en publicaciones de las universidades estatales costarricenses (top 6), como autores principales. De manera agregada es preciso señalar que Costa Rica cuenta con presencia en todos los continentes. 


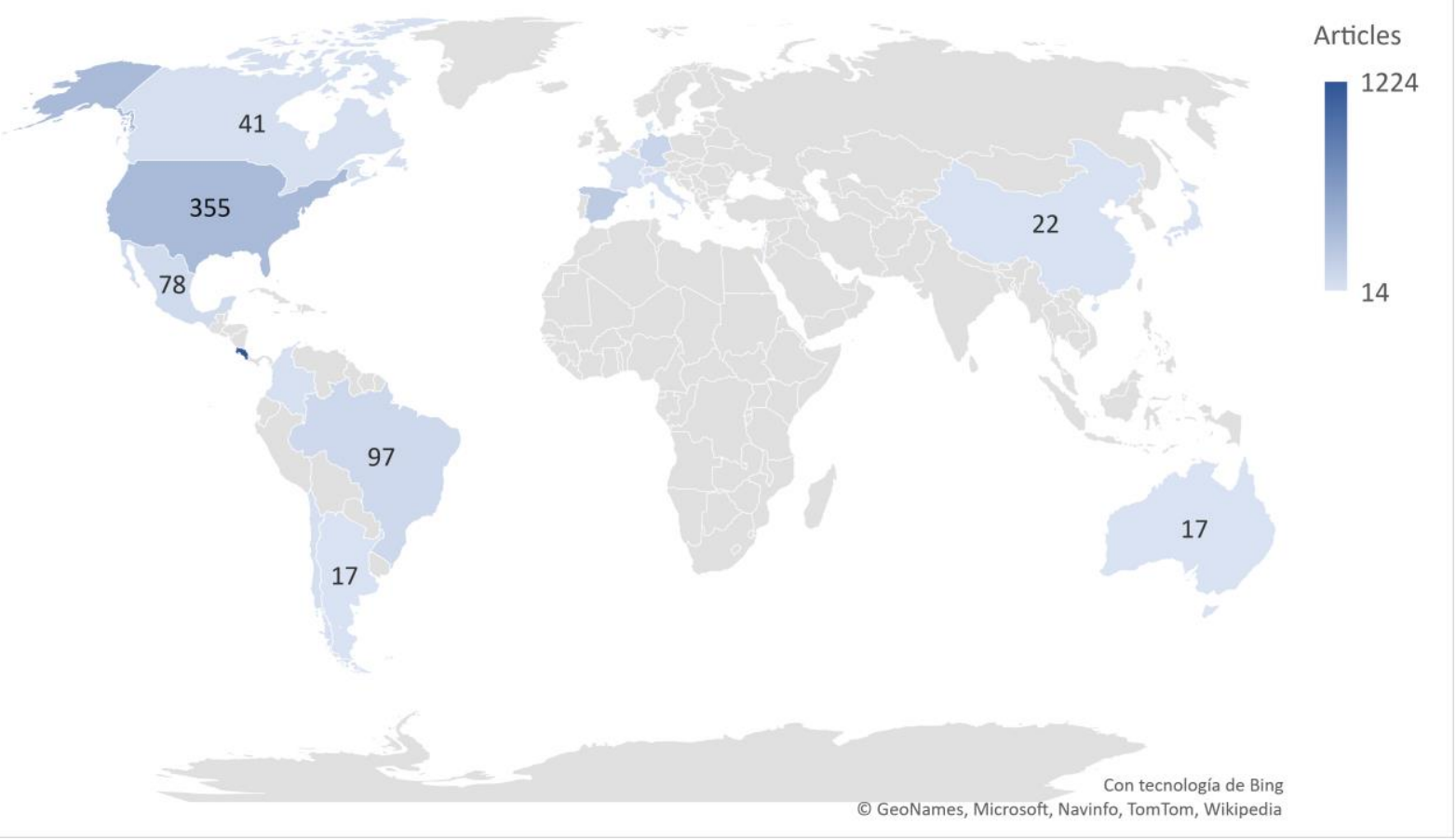

Figura 8. Mapa de calor sobre la colaboración en publicaciones de las universidades estatales costarricenses como autores principales, por país de afiliación, (2011-2019)

Fuente: Elaboración propia con datos de Scopus (2011-2019).

Entre las principales afiliaciones institucionales con las cuales han colaborado o participado las universidades estatales de Costa Rica, se destaca la Universidad de California en primer lugar con 266 publicaciones en colaboración, la Universidad de Ciencias Médicas de Teherán con 217 publicaciones, la Universidad Nacional Autónoma de México con 133 colaboraciones, entre otras. También se destaca dentro de un top 10 universidades como Harvard y Oxford.

\section{c. Características de consumo científico y citación de la producción científica costarricense de las universidades estatales indexada en Scopus, 2011-2019}

En este apartado se analizarán algunos indicadores relacionados con el consumo científico, es decir, indicadores vinculados con el uso de la producción científica en términos de citas, visibilidad e impacto (medido con el índice $\mathrm{H}$ ). 
De acuerdo con los datos analizados de las publicaciones en estudio, entre las revistas más citadas se destacan en el primer puesto la revista Toxicon de cuartil 3 de impacto en toxicología, con 3.680 citaciones, con 2.259 citaciones se posiciona en el segundo lugar la revista Biología Tropical (cuartil 2 en ciencias biológicas) y, en el tercer lugar, la revista Science con 1.813 citaciones, posicionada en el cuartil de impacto 1. En la figura 9 se muestra un top de las revistas más citadas.

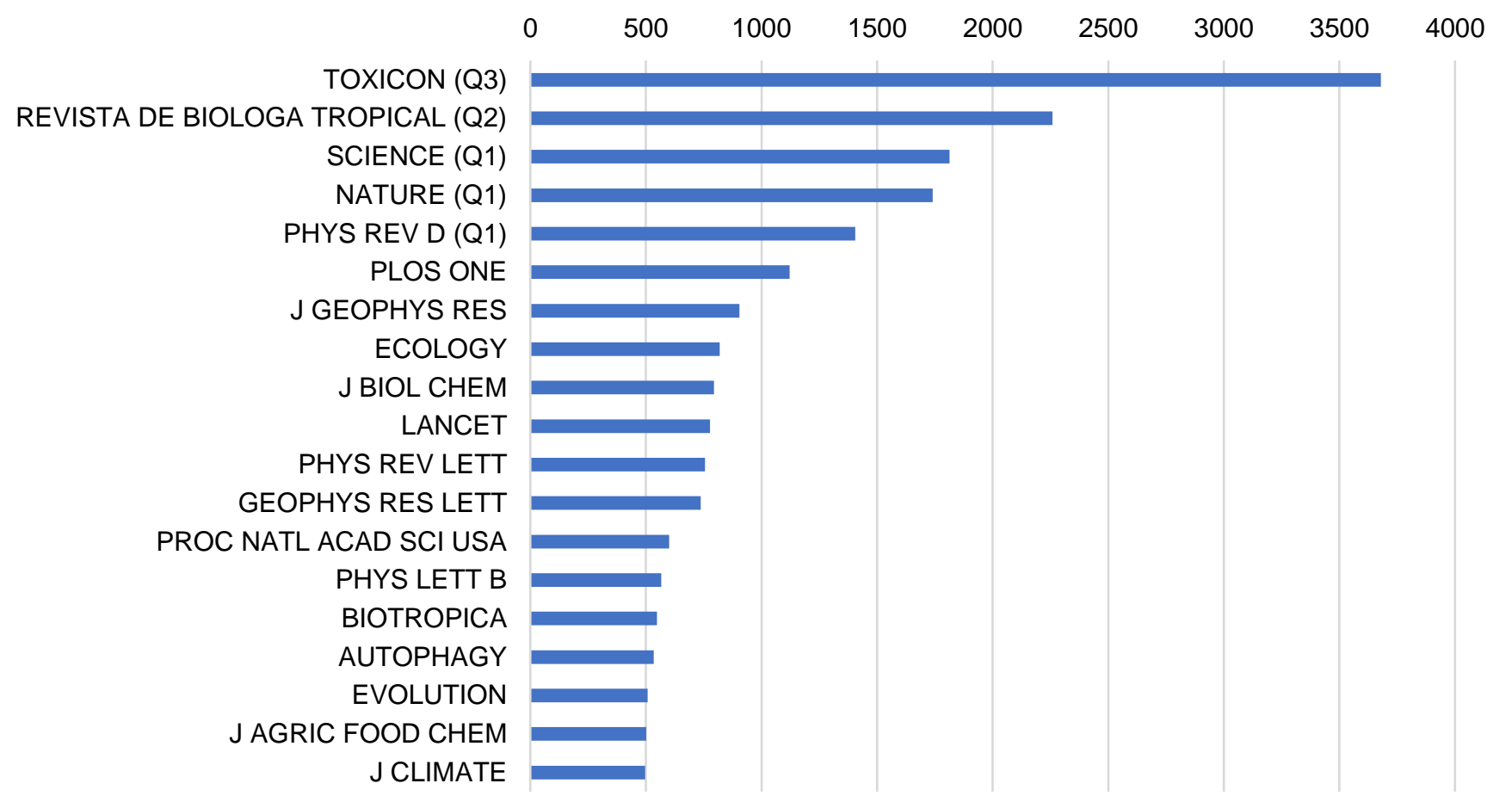

Figura 9. Citas recibidas por revista, 2011-2019

Fuente: Elaboración propia con datos descargados de Scopus, 2011-2019

Por otra parte, al evaluar las revistas tomando en cuenta el impacto, es importante mencionar que tanto la productividad, como la citación y el impacto son tres elementos distintos. El impacto suele medirse con otros tipos de indicadores, por ejemplo, el índice $\mathrm{H}$ de publicación. Este índice está construido o toma en cuenta tanto la productividad (número de publicaciones) como la citación, también es necesario considerar que el índice $\mathrm{H}$ es sumamente variable con el tiempo, una cita puede variar de un día a otro, asimismo, deja de ser sensible a mayor citación ya que puede quedar estático. Por tanto, este apartado lo que busca es demostrar el comportamiento de este indicador, sin realizar una comparación entre disciplinas diferentes, entre otros. 
Según lo anterior, con mayor impacto (en términos de su índice $\mathrm{H}$ ) se posiciona la revista Journal of Proteomics (Q1) con un índice $\mathrm{H}$ de 24. Es importante recordar que para esta revista en total se contabilizaron 38 artículos publicados, por parte de las universidades estatales costarricenses, mientras que para la revista que ocupa el segundo puesto (Plos One (Q1)), se contabilizaron 86 artículos con un índice $\mathrm{H}$ de 23. Estos números se traducen en, por ejemplo, para Journal of Proteomics al menos 24 artículos de las universidades estatales costarricenses, han sido citados al menos 24 veces durante el tiempo de referencia.

En el tercer y cuarto puesto se ubican la revista Toxicon (Q3) y la revista The Lancet (Q1). En cuadro 2 se detalla las publicaciones de las universidades estatales de Costa Rica con mayor impacto por revista.

\section{Cuadro 2}

Publicaciones con mayor impacto por revista (top 10), 2011-2019

\begin{tabular}{lccc}
\hline Source & índice H & $\begin{array}{c}\text { Citación } \\
\text { total }\end{array}$ & $\begin{array}{c}\text { Número de } \\
\text { publicaciones }\end{array}$ \\
\hline JOURNAL OF PROTEOMICS & 24 & 1378 & 38 \\
\hline PLOS ONE & 23 & 1531 & 86 \\
\hline TOXICON & 20 & 1381 & 83 \\
\hline THE LANCET & 20 & 13036 & 22 \\
\hline REVISTA DE BIOLOGIA TROPICAL & 14 & 986 & 248 \\
\hline PLOS NEGLECTED TROPICAL DISEASES & 13 & 418 & 19 \\
\hline TOXINS & 12 & 383 & 23 \\
\hline FOOD RESEARCH INTERNATIONAL & 12 & 776 & 16 \\
\hline HYDROLOGICAL PROCESSES & 11 & 279 & 20 \\
\hline GEOCHEMISTRY, GEOPHYSICS, GEOSYSTEMS & 10 & 384 & 16 \\
\hline SCIENCE OF THE TOTAL ENVIRONMENT & 9 & 310 & 13 \\
\hline PROCEEDINGS OF THE NATIONAL ACADEMY OF & 9 & 355 & 10 \\
SCIENCES OF THE UNITED STATES OF AMERICA & & & \\
\hline ZOOTAXA & 8 & 259 & 52 \\
\hline SCIENTIFIC REPORTS & 8 & 315 & 29 \\
\hline JOURNAL OF VOLCANOLOGY AND & 8 & 178 & 16 \\
GEOTHERMAL RESEARCH & 8 & 251 & 14 \\
\hline FRONTIERS IN MICROBIOLOGY & 8 & 138 & 14 \\
\hline JOURNAL OF FOOD PROTECTION & 8 & 222 & 13 \\
\hline NATURE COMMUNICATIONS & & & \\
\hline
\end{tabular}




\begin{tabular}{llll}
\hline TICKS AND TICK-BORNE DISEASES & 8 & 139 & 12 \\
\hline ENVIRONMENTAL SCIENCE AND POLLUTION & 8 & 132 & 10 \\
RESEARCH & & \\
\hline
\end{tabular}

Fuente: Elaboración propia con datos descargados de Scopus 2011-2019.

También resulta importante destacar algunos de los artículos con mayor citación. De igual manera constituyen indicadores de consumo científico; en el primer lugar destaca el artículo de review titulado: "Guidelines for the use and interpretation of assays for monitoring autophagy (3rd edition)" publicado en el año 2016 en la revista internacional Autophagy de cuartil Q1 de impacto de acuerdo con datos del SCImago Journal and Country Rank, además, esta publicación cuenta con 2.731 citaciones totales a setiembre del 2020.

En el segundo puesto, con mayor citación, se ubica "Guidelines for the use and interpretation of assays for monitoring autophagy" artículo tipo review publicado en el año 2012, en la revista Autophagy con una citación total de 2.488 a setiembre del año 2020. El tercer documento más citado para el período de referencia es el artículo titulado "Global, regional, and national life expectancy, all-cause mortality, and cause-specific mortality for 249 causes of death, 19802015: a systematic analysis for the Global Burden of Disease Study 2015" publicado en el año 2016 en la revista de salud The Lancet con un total de 2.414 citaciones. Esta revista actualmente se posiciona en el cuartil Q1 dentro de la disciplina de las ciencias médicas.

A continuación (ver Cuadro 3) se presenta el detalle de los 10 artículos más citados que forman parte de la producción científica realizada por las universidades estatales costarricenses, para los años en estudio. Tal y como se puede observar existe una predominancia en términos de citación de artículos muy especializados, en este caso se destaca la presencia de mayor citación en artículos publicados en la revista de medicina internacional The Lancet. 


\section{Cuadro 3}

Artículos con mayor citación, 2011-2019

\begin{tabular}{|c|c|c|c|c|}
\hline $\mathbf{N}^{\circ}$ & Título del document & Año & $\begin{array}{l}\text { Fuente de } \\
\text { publicación }\end{array}$ & Total Citations \\
\hline 1 & $\begin{array}{l}\text { Guidelines for the use and interpretation of } \\
\text { assays for monitoring autophagy (3rd edition) }\end{array}$ & 2016 & Autophagy & 2731 \\
\hline 2 & $\begin{array}{l}\text { Guidelines for the use and interpretation of } \\
\text { assays for monitoring autophagy }\end{array}$ & 2012 & Autophagy & 2488 \\
\hline 3 & $\begin{array}{l}\text { Global, regional, and national life expectancy, } \\
\text { all-cause mortality, and cause-specific mortality } \\
\text { for } 249 \text { causes of death, 1980-2015: a } \\
\text { systematic analysis for the Global Burden of } \\
\text { Disease Study } 2015\end{array}$ & 2016 & The Lancet & 2414 \\
\hline 4 & $\begin{array}{l}\text { Global, regional, and national incidence, } \\
\text { prevalence, and years lived with disability for } \\
310 \text { diseases and injuries, 1990-2015: a } \\
\text { systematic analysis for the Global Burden of } \\
\text { Disease Study } 2015\end{array}$ & 2016 & The Lancet & 2412 \\
\hline 5 & $\begin{array}{l}\text { Global, regional, and national comparative risk } \\
\text { assessment of } 79 \text { behavioural, environmental } \\
\text { and occupational, and metabolic risks or } \\
\text { clusters of risks, 1990-2015: a systematic } \\
\text { analysis for the Global Burden of Disease } \\
\text { Study } 2015\end{array}$ & 2016 & The Lancet & 1550 \\
\hline 6 & $\begin{array}{l}\text { Global, regional, and national incidence, } \\
\text { prevalence, and years lived with disability for } \\
354 \text { Diseases and Injuries for } 195 \text { countries } \\
\text { and territories, } 1990-2017: \text { A systematic } \\
\text { analysis for the Global Burden of Disease } \\
\text { Study } 2017\end{array}$ & 2018 & The Lancet & 1000 \\
\hline 7 & $\begin{array}{l}\text { Global, regional, and national disability- } \\
\text { adjusted life-years (DALYs) for } 315 \text { diseases } \\
\text { and injuries and healthy life expectancy } \\
\text { (HALE), 1990-2015: a systematic analysis for } \\
\text { the Global Burden of Disease Study } 2015\end{array}$ & 2016 & The Lancet & 899 \\
\hline 8 & $\begin{array}{l}\text { Improved allometric models to estimate the } \\
\text { aboveground biomass of tropical trees }\end{array}$ & 2014 & $\begin{array}{l}\text { Global } \\
\text { Change } \\
\text { Biology }\end{array}$ & 828 \\
\hline
\end{tabular}




\begin{tabular}{|l|l|l|l|l|}
\hline & $\begin{array}{l}\text { Global, regional, and national age-sex-specific } \\
\text { mortality for 282 causes of death in 195 } \\
\text { countries and territories, 1980-2017: a } \\
\text { systematic analysis for the Global Burden of } \\
\text { Disease Study 2017 }\end{array}$ & The Lancet & 796 \\
\hline 10 & $\begin{array}{l}\text { Global, regional, and national disability- } \\
\text { adjusted life-years (DALYs) for 333 diseases } \\
\text { and injuries and healthy life expectancy (HALE) } \\
\text { for } 195 \text { countries and territories, 1990-2016: A } \\
\text { systematic analysis for the Global Burden of } \\
\text { Disease Study 2016 }\end{array}$ & The Lancet & 723 \\
\hline
\end{tabular}

Fuente: Elaboración propia con datos descargados de Scopus, 2011-2019.

\section{Conclusiones y recomendaciones}

La producción científica de Costa Rica, por parte de sus universidades estatales, indexada en este índice ha crecido en promedio un 13\%. Al año 2019 se han sumado a Scopus 6 revistas costarricenses pertenecientes a la Universidad de Costa Rica y a la Universidad Nacional.

Las universidades estatales de Costa Rica han incrementado su inversión realizada en I+D, especialmente para los años 2015-2017, según datos de Vílchez et al. (2019) y Vílchez et al. (2020). Este hecho se ha visto reflejado en un aumento de los proyectos de investigación y publicaciones para esos años. Con estos esfuerzos e incremento en el acervo del conocimiento, se refuerza la necesidad de monitorear la investigación como parte de la rendición de cuentas y la detección de oportunidades de mejora para incrementar la calidad de la investigación.

También es importante recordar que la producción científica analizada en este documento no constituye el total de la producción científica y académica realizada por las universidades estatales de Costa Rica. Sin embargo, a nivel de la base de datos internacional Scopus las universidades estatales publican en promedio un $66 \%$ de toda la producción costarricense que se encuentra indexada en ella. También conviene tener en cuenta que, aunque una revista determinada no forme parte de Scopus, no significa que la revista no posea impacto, 
visibilidad o calidad según lo exponen de Rozemblum, et al. (2020). Muchas revistas que quizás no forman parte de Scopus, pueden poseer gran desempeño en indicadores relacionados con la calidad, la visibilidad, entre otros.

La producción científica analizada ha demostrado que Costa Rica ha ganado gran diversificación de temáticas e incursión en distintas áreas de la ciencia como lo son biología, medicina, veterinaria, química, computación y algunas que poseen carácter multidisciplinar como, por ejemplo, la neurociencia. Asimismo, referente a la colaboración internacional se logró demostrar que Costa Rica y sus universidades estatales, no únicamente tienen presencia en todos los continentes, sino que mayoritariamente figura un investigador costarricense como principal, coordinador o líder de estas investigaciones, lo cual puede ser una plataforma e impulso para lograr fortalecer redes de investigación a nivel internacional.

Es importante que las autoridades universitarias tengan en cuenta que el idioma inglés es fundamental para comunicar ciencia, ya que es el idioma que prevalece, además, hay gran colaboración con universidades e institutos de Estados Unidos. Por lo tanto, cada vez es más importante que los investigadores se formen en el idioma inglés y perfeccionen sus habilidades de escritura científica. También se recomienda orientar a los investigadores en la publicación de diversos tipos documentales como, por ejemplo, libros, artículos de revisión, entre otros, los cuales son productos potenciales para la publicación en muchas revistas indexadas en el índice Scopus.

Por otra parte, es conveniente monitorear las relaciones de colaboración con institutos, escuelas, universidades $u$ organizaciones que tienen participación en publicaciones costarricenses, para fortalecer y mejorar las relaciones académicas de bilateralidad y cooperación internacional, la colaboración en investigación es el primer paso para abrir una gama de oportunidades para Costa Rica, especialmente, su talento humano y avances científicos e investigativos. 
Las universidades deben emprender la tarea de identificar a investigadores experimentados, por ello las posibles redes de coautoría y colaboración es fundamental, esto puede servir como insumo para proponer e impulsar a investigadores más jóvenes y con menor experiencia a introducirse en estos grupos, orientados por estos investigadores con mayor trayectoria. De igual forma, al identificar estos investigadores y el tipo de trabajo que realizan, así como los productos que desarrollan, puede pensarse en estrategias para vincularlos al sector productivo y aportar soluciones desde la academia.

Es necesario que las autoridades en materia de educación superior estatal brinden monitoreo y seguimiento a los productos derivados de las actividades de $I+D$, ya que se consideran pilares fundamentales en el desarrollo y en la fundación de las sociedades basadas en el conocimiento, asimismo, identificar el potencial dentro de las mismas universidades para responder a los retos que se presentan en la sociedad.

\section{Referencias}

Alarcon, C., Diaz, M., Vera, V., Alva, C., y Metcalf, T. (2019). A Bibliometric Analysis of the Latin American Research on Stroke 2003-2017. World Neurosurgery, 129, e545-e554. https://doi.org/10.1016/j.wneu.2019.05.212

Aria, M., y Cuccurullo, C. (2017). Bibliometrix: An R-tool for comprehensive science mapping analysis. Journal of Informetrics, 11 (4), 959-975

Cortés, D. (2007). Medir la producción científica de los investigadores universitarios: la bibliometría y sus límites. Revista de La Educación Superior, (2) (142), 43-65.

Duran, E., Astroza, K., Ocaranza, J., Peñailillo, D., Pavez, I., y Ramírez, R. (2019). Scientific Research on Nanotechnology in Latin American Journals Published in SciELO: Bibliometric Analysis of Gender Differences. NanoEthics, 13 (2), 113-118. https://doi.org/10.1007/s11569-019-00344-5

Gómez, I., y Bordons, M. (2009). Limitaciones en el uso de los indicadores bibliométricos para la evaluación científica.

Hirsch, J. E. (2010). An index to quantify an individual's scientific research output that takes into account the effect of multiple coauthorship. Scientometrics, 85, 741. 
Hirsch, J.E. (2005). An index to quantify an individual's scientific research output that takes into account the effect of multiple coauthorship, Department of Physics, University of California.

Lascurain, M. L. (2006). La evaluación de la actividad científica mediante indicadores bibliométricos. Bibliotecas, 24(1 y 2), 9-26.

Lomonte, B., y Ainsworth, S. (2002). Publicaciones científicas de Costa Rica en el Science Citation Index: Análisis bibliométricos del trienio 1999-2001. Revista de Biología Tropical, 50(3-4), 951-962.

Macias, C. (1998). Papel de la informetría y de la cienciometría y su perspectiva. 35-41.

Microsoft (n.d). Guardar un libro en formato de texto (. txt o. csv). Recuperado el 16 de agosto 2021 de https://support.microsoft.com/es-es/office/guardar-un-libro-en-formato-de-textotxt-o-csv-3e9a9d6c-70da-4255-aa28-fcacf1f081e6

Montazerian, M., Dutra, E., y Eckert, H. (2020). Prolificacy and visibility versus reputation in the hard sciences. Scientometrics. https://doi.org/10.1007/s11192-020-03369-w

Nielsen, V., Azofeifa, A. B., y Monge, J. (2012). Bibliometry of Costa Rica biodiversity studies published in the revista de biología tropical/international journal of tropical biology and conservation (2000-2010): The content and importance of a leading tropical biology journal in its 60th anniversary. Revista de Biologia Tropical, 60(4), 1405-1413. https://doi.org/10.15517/rbt.v60i4.2043

Ramírez, G. (2020). Publicaciones [Correo electrónico].

Rozemblum, C., Alperin, J. P., y Unzurrunzaga, C. (2020). Las limitaciones de Scopus como fuentes de indicadores: Buscando una visibilidad integral para revistas argentinas en ciencias sociales. Zenodo. http://doi.org/10.5281/zenodo.4110847

Sancho, R. (2003). Indicadores bibliométricos utilizados en la evaluación de la ciencia y tecnología. Revisión bibliográfica. Inteligencia Competitiva, 1, 9-5. https://doi.org/10.5209/rev_UNIS.2003.n1.29484

Sanz, E., y Martín, C. (1997). Técnicas bibliométricas aplicadas a los estudios de usuarios. Revista General de Información y Documentación, 7 (2), 41-68. https://doi.org/10.5209/RGID.11724

SCImago, (n.d.). SJR - SCImago Journal \& Country Rank [Portal]. Recuperado mayo 2020, 
from http://www.scimagojr.com

Spinak, E. (1998). Indicadores Cienciométricos. Artigos, 141-148. https://doi.org/10.1590/s0100-

Uceda, L. G. (1997). Teoría de la ciencia, Documentación y Bibliometría. Revista General de Información y Documentación, 7(2).

Urbizagástegui, R. (2014). Estudio sincrónico de obsolescencia de la literatura: El caso de la Ley de Lotka. Investigación Bibliotecológica, 28 (63), 85-113. https://doi.org/10.1016/s0187-358x(14)72577-8

Vílchez Moreira, M., [et al.] (2020). Indicadores de la Investigación Universitaria 2014-2018. San José, C.R.: CONARE, OPES

Vílchez Moreira, M. [et al.] (2019). Indicadores de la investigación universitaria 2013 - 2017. San José, C.R.: CONARE, OPES.

Yılmaz, B., Dinçol, M. E., y Yalçın, T. Y. (2019). A bibliometric analysis of the 103 top-cited articles in endodontics. Acta Odontologica Scandinavica, 77 (8), 574-583. https://doi.org/10.1080/00016357.2019.1621378

\section{Notas de las personas autoras}

* Este artículo se deriva del proyecto de investigación "Análisis bibliométrico de la producción científica indexada en la base de datos internacional Scopus, publicada por las cinco universidades públicas de Costa Rica, 2011-2019", cuyo informe final de investigación puede encontrarse en el siguiente enlace: http://hdl.handle.net/20.500.12337/8066

1 "La infometría es el estudio de los aspectos cuantitativos de la información en cualquier forma, no solo a partir de registros catalográficos o bibliografía, y abarca a cualquier grupo social por lo que no se limita sólo al científico" (Macias, 1998, p.36).

2 "Los archivos csv. corresponden a un archivo de texto delimitado por comas" Microsoft (n.d).

Silvia Sáenz León, Investigadora. Economista, Bachiller. Consejo Nacional de Rectores, Costa Rica. Correo electrónico: ssaenz@conare.ac.cr. ORCID: https://orcid.org/0000-0003-0801$\underline{8224}$

Nanci Rodríguez Ramos, Investigadora. Economista, Licenciada. Consejo Nacional de Rectores, Costa Rica. Correo electrónico: nrodriguez@conare.ac.cr. ORCID: https://orcid.org/0000-0002-7848-4434 